\title{
GA-MIP: Genetic Algorithm based Multiple Mobile Agents Itinerary Planning in Wireless Sensor Networks
}

\author{
${ }^{1}$ Wei Cai, ${ }^{1}$ Min Chen, ${ }^{2}$ Takahiro Hara, ${ }^{2,3}$ Lei Shu \\ ${ }^{1}$ School of Computer Science and Engineering, Seoul National University, Republic of Korea \\ ${ }^{2}$ Department of Multimedia Engineering, Osaka University, Japan \\ ${ }^{3}$ Digital Enterprise Research Institute, National University of Ireland, Galway \\ caiwei@mmlab.snu.ac.kr, minchen@snu.ac.kr, hara@ist.osaka-u.ac.jp, lei.shu@ieee.org
}

\begin{abstract}
It has been proven recently that using Mobile Agent (MA) in wireless sensor networks (WSNs) can drastically help to obtain the flexibility of application-aware deployment. Normally, in any MA based sensor network, it is an important research issue to find out an optimal itinerary for the MA in order to achieve efficient and effective data collection from multiple sensory data source nodes. In this paper, we firstly investigate a number of conventional single MA itinerary planning based schemes, and then indicate some shortcomings of these schemes, since only one MA is used by them. Having these investigations and analysis, a novel genetic algorithm based multiple MAs itinerary planning (GA-MIP) scheme is proposed to address the shortcomings of large latency and global unbalancing of using single MA, and its effectiveness is proved by conducting the extensive experiments in professional environment.
\end{abstract}

\section{INTRODUCTION}

A wireless sensor network (WSN) [1] consists of spatially distributed autonomous sensors to cooperatively monitor physical or environmental conditions, such as temperature, sound, vibration, pressure, motion or pollutants. The development of WSNs was motivated by military applications, such as battlefield surveillance. They are now used in many industrial and civilian application areas, including industrial process monitoring and control, machine health monitoring, environment and habitat monitoring, healthcare applications, home automation, and traffic control.

Mobile Agent (MA) system [2] can cope with situationaware applications [3] [4], e.g., habitat monitoring and medical care, in WSNs [5] [6]. As a special kind of software, MA migrates among network nodes to carry out task(s) autonomously, e.g., collecting sensory data from a number of source nodes, and flexibly to handle network dynamics, in order to achieve the specific requirements of the agent dispatcher (i.e., the sink node). MA system has been proven to be an efficient approach to enhance such capabilities of WSNs [7] [8].

Normally, the MA design in WSNs can be decomposed into four components [5], i.e., 1) architecture, 2) itinerary planning, 3) middleware system design and 4) agent cooperation. Among these four components, itinerary planning determines the order of sensory data source nodes to be visited during the MA migration, which has a significant impact on the performance of the MA systems. Thus, find out an optimal itinerary for the MA to visit a number of source nodes is critical. However, finding an optimal itinerary had already been proven to be NP-hard [9], generally heuristic algorithms are proposed and applied to compute competitive itineraries with sub-optimal performance.

A number of itinerary planning schemes have been proposed in recent researches [7] [8] [9] [10], but most of them focus only on the single MA problem in WSNs. Although, using MA in WSNs can help to obtain the flexibility of applicationaware deployment, sometimes, using only single MA in a WSN can also bring some visible shortcomings, e.g., the long latency and the global unbalancing. In order to address these shortcomings of using only single MA, multiple MAs itinerary planning is then proposed in [11]. Following the same approach, but different idea, in this paper, a novel genetic algorithm (GA) based multiple MAs itinerary planning (GAMIP) scheme is proposed, which mainly aims at optimizing the number of MAs and planning an efficient itinerary for each MA.

To realize the GA-MIP algorithm, we encode the Source Node Sequence and the Source Node Group (see section IV-C) into numbers as the genes for genetic evolution. First, we set up a searching space filled with randomly selected genes. Then, we perform an iterative evolution approach. In each iteration, evolution operators such as crossover and mutations are applied to increase the variety of the genes. After these procedures, the selection operator selects the better genes to survive for the next generation, which is analogous to the natural-selection in the real world. After a number of evolution iterations, the solution corresponding to an efficient strategy of itinerary planning will be obtained.

The scientific research contributions of this research work include the following points:

- Novelty: To the best of our knowledge, this research work is the first effort that try to solve the MIP problem based on the genetic algorithm.

- Optimization: Different from previous MIP solution [11], which divide the MIP solution into 3 components: 1) finding the optimal number of MAs 2) grouping source 
nodes for MAs 3) determining the visiting sequence for each MA, the proposed GA-MIP scheme consider finding the optimal number of MAs, subsets for source nodes and the visiting sequence for each MA as ONE problem. It provides sub-optimal solution with shorter task duration and lower communication cost while the computational complexity is not relevant to the number of source nodes.

The reminder of the paper is organized as following: Related work is introduced in Section II. Then we describe the novel genetic algorithm based multiple mobile agents itinerary planning scheme in Section IV. Simulation analysis are performed in Section V. Finally, Section VI concludes this paper.

\section{RELATED WORK}

A number of researches have been conducted for MA itinerary planning in WSNs [7] [8] [9] [10] [12] [11]. Among these previous heuristic proposals, Local Closest First (LCF) and Global Closest First (GCF) are the simplest approaches [8] for MA itinerary planning. LCF searches for the next sensory data source node with the shortest distance to the current node while GCF selects the closest node to the sink node as its next source node. MADD [7] is similar with LCF but selects the farthest source node as the starting point of the itinerary. All of these three approaches are not energy efficient as indicated in [9], in which the authors propose a better scheme named IEMF ${ }^{1}$. IEMF extends LCF by considering estimated communication cost, and achieves further reduction on energy consumption by choosing the first source node to be visited according to the estimated communication cost, since choosing choosing different first source nodes results in different total communication cost. In [10], a genetic algorithm based mobile itinerary planning is proposed to exploit the global information of sensor detection signal levels and link power consumption.

However, all of these researches only focus on the single MA itinerary planning (SIP) problem. Thus, they have some intrinsic shortcomings of using single MA, especially when the number of source nodes is large [12], e.g., 1) Delay Issue: extensive delay is needed when a single agent works for a network consisting of hundreds of or even thousands of sensor nodes; 2) Traffic Load Issue: in the perspective of the whole network, the traffic load is put on the nodes along the single flow. Therefore, sensor nodes traversed in the agent itinerary will deplete their energy much quicker than other nodes. This drawback will reduce the lifetime of the network ${ }^{2}$.

To address these problems, a multiple mobile agents itinerary planning (MIP) is proposed in [11], in which the authors discover a dense center of source nodes and then organize the sources nodes in a predefined radius $R$ as a group

\footnotetext{
${ }^{1}$ Later, IEMF is extended to IEMA by an iterative approach.

${ }^{2}$ There is no universally agreed definition of network lifetime as it depends on the specific application. The lifetime can be measured by the time when the first node exhausts its energy, or when a certain fraction of nodes are dead, or even when all nodes are dead. Alternately, it may be reasonable to measure the network lifetime by application-specific parameters, such as the time when the network can no longer relay sensory data packets. In this paper, we define WSNs lifetime as the time when the first node dies due to energy depletion.
}

for one MA. This procedure repeats until all source nodes are visited. However, this paper still leaves an open research issue on choosing the optimal number of source nodes, in order to minimize the total communication cost.

In this paper, the proposed GA-MIP scheme solves the MIP problem in an unconventional approach, in which it does not set the number of MAs and the visit sequence for MAs in particular rules in advance, but applies an genetic evolution approach to search a sub-optimal solution based on the estimated cost.

\section{Network Model And Problem Statement}

\section{A. Network Model}

The considered WSN in this paper consists a number of densely and randomly deployed static sensor nodes, as shown in the Fig. $1^{3}$. A static sink node is deployed in the central location of the sensor network, with infinite power supply and strong computational capability. A number of sensory source nodes are randomly deployed in the WSN. All the sensor nodes have the uniform transmission radius, and any two directly connected (1-hop) sensor nodes have the stable bi-directional communication. The degree of the sensor nodes deployment density can guarantee that each sensor node has at least two 1-hop neighbor nodes. A number of MAs can be issued by the sink node to visited the randomly deployed source nodes simultaneously with different itineraries, in which each MA has its own itinerary for visiting a subset of the total source nodes.

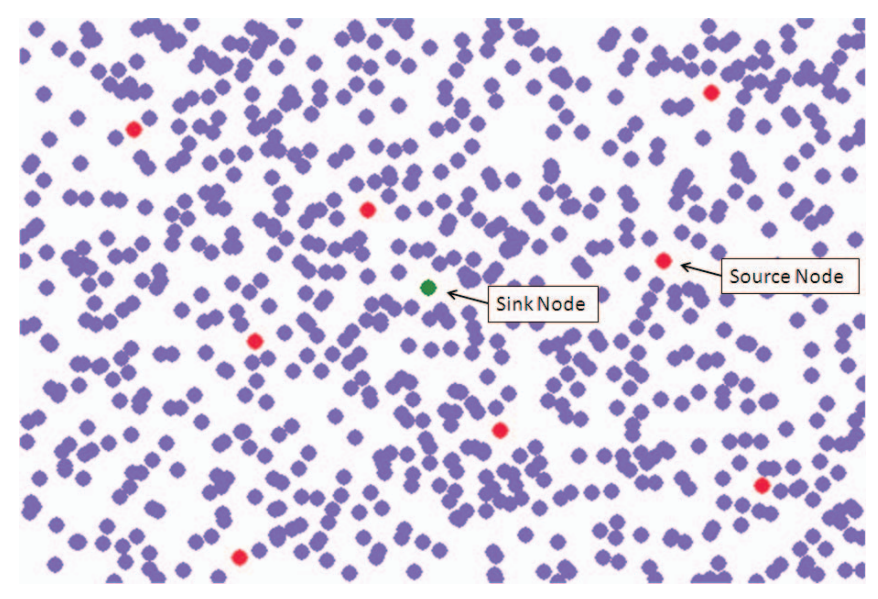

Fig. 1. An Example of the Studied Network Model

\section{B. Problem Statement}

This paper is a further extension work of the [11], the major research problem is to find the optimal number of MAs and subsets of all source nodes to minimize the task duration and total communication cost.

\footnotetext{
${ }^{3}$ This figure is produced by using NetTopo [13].
} 


\section{Genetic Algorithm based Multi-Mobile Agents Itinerary PlanNing}

\section{A. Introduction to Genetic Algorithm}

Genetic Algorithm [14] (GA) is adaptive heuristic search algorithm based on the evolutionary theory of genetic and natural selection, which will produce the fittest survival. In a GA system, each solution to the problem was described as an individual with genetic information in the nature. The solutions produce children that inherit mixture characteristics from their parents. Meanwhile, an opportunistic mutation may happen to generate new individuals. Through the evaluation by a fitness function, the better individuals could survive. As time goes on, the survivals contain the excellent genes which represent the better solutions to the problem.

\section{B. The GA-MIP Scheme}

In this section, we introduce the GA-MIP scheme. The encoding methods are explained in Section IV-C, and Section IV-D introduces the operators for the evolution.

Definition 1. Code of Source Node Sequence. The Code of Source Node Sequence is an array of the source nodes' identifiers, which implies the order for a MA to visit the corresponding source nodes. One MA can have only one final Code of Source Node Sequence for its visiting itinerary.

Definition 2. Code of Source Node Group. The Code of Source Node Group is an array of integer numbers, in which each number indicates the number of source nodes that are allocated in the corresponding Source Node Group.

\section{Encoding Method}

To enable GA implementation, we must present a solution to the problem as a gene. In GA-MIP, a gene consists of two parts: (1) Code of Source Node Sequence (sequence array); (2) Code of Source Node Group (group array). Note that the combination of sequence array and group array represents one itinerary planning solution.

Fig.2 shows an illustrative example for the realization of their encodings. We denote the output of Source Node Sequence encoding by sequence array, and denote the output of Source Node Group encoding by group array. The number of non-zero elements in group array represents the number of MAs. The value of each non-zero element denotes the number of source nodes to be visited by the corresponding MA. In this figure, the three non-zero elements (i.e., 4,3,1) in the group array correspond to three MAs. The first MA (MA1 in Fig.2) will visit 4 source nodes in the order of 6, 3, 2, 4; MA2 will visit the source nodes in the order of $8,1,7$; and MA3 will visit the source node with ID of 5.

Theorem 1. The proposed encoding mechanism in GAMIP scheme ensures each combination of sequence array and group array can only represent one unique MIP solution. Proof: It is proved in Genetic Theory [14].

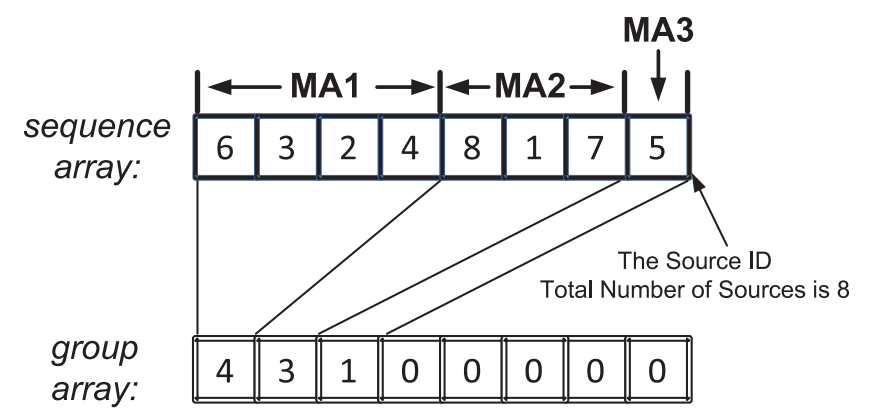

Fig. 2. The Encoding Example with 3 MAs and 8 Source Nodes

Since each group array is associated with a sequence array, the elements in the group array should be sorted by a numerical order. Otherwise, the combinations of the two arrays likely produce repetitive solutions. Additionally, it is easy to perform crossover and mutation.

\section{Operators}

In this section, we describe the genetic operators for the GA-MIP scheme. As conventional operators for GA, we implemented the crossover, mutation and selection operators.

1) Crossover Operators: Crossover operator is an essential operator in GA. It imitates the way of natural biological evolution. In previous GA works, there are several crossover schemes have been proposed, such as one-point crossover [15] and multi-point crossover [16]. However, we have to find out a proper way of crossover to inherit the better genes in the search space for our evaluation criterion.

In our approach, crossover is only applied between the sequence arrays attach with the same group array as shown in Fig.3. We first randomly select a non-zero element (denoted by $m$ ) in group array. Corresponding to its associated sequence array, $m$ is mapping to a sequence segment (see Fig.2). The two sequence segments which are located in the same positions in the two sequence arrays will be exchanged each other in our crossover procedure. ${ }^{4}$

As an example show in Fig.3, we assume that element "3" in group array was selected as the indicator of the crossover section. Thus, the segment "1-6-8" in sequence array 1 is inserted into the associated position of sequence array 2, vise versa. This operation produces two arrays with 12 elements, which we call it "Internal Results" in the figure. Apparently, the they are invalid since they include duplicate elements (e.g., nodes 6, 7, 9 in sequence array 1, nodes 1, 6, 8 in sequence array 2 as shown in Fig.3(b)). Thus, the elimination procedure is required for producing the final children arrays.

2) Mutation Operators: The mutation operator is used to keep the variety of the genes so that the discovery of new solutions is possible. In our approach, both sequence array mutation and group array mutation are implemented.

For the sequence array, the operator randomly selects two elements in the array and switches their positions. For the

\footnotetext{
${ }^{4}$ Note that duplicate and lost in genes happened during the crossover operations should be repaired to ensure the correctness of the sequence code.
} 
Parents:

\begin{tabular}{rl|l|l|l|l|l|l|l|l|l||} 
sequence array 1: & 2 & 3 & 5 & 4 & 1 & 6 & 8 & 7 & 9 \\
group array 1: & \multicolumn{1}{|c||}{4} & 3 & 2 & 2 & 0 & 0 & 0 & 0 & 0 & 0 \\
\hline
\end{tabular}

\section{Internal Results:}

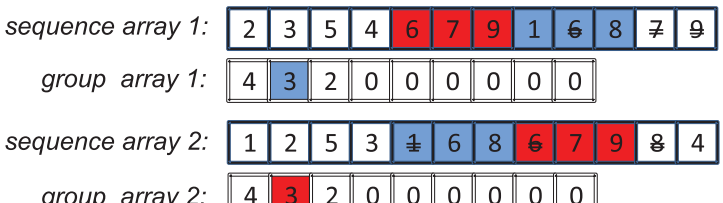

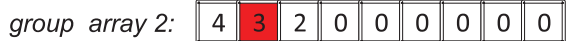

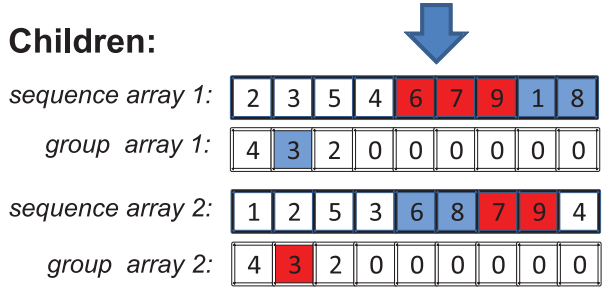

Fig. 3. The Example for Sequence Crossover

group array, similar operation is performed that a non-zero element with larger value will be decreased by 1 and a smaller element will be increased by 1 . Note that we have to guarantee the elements in group array is in numerical order, thus a sort for the group array should be performed after the random mutation.

\section{Before Mutation:}

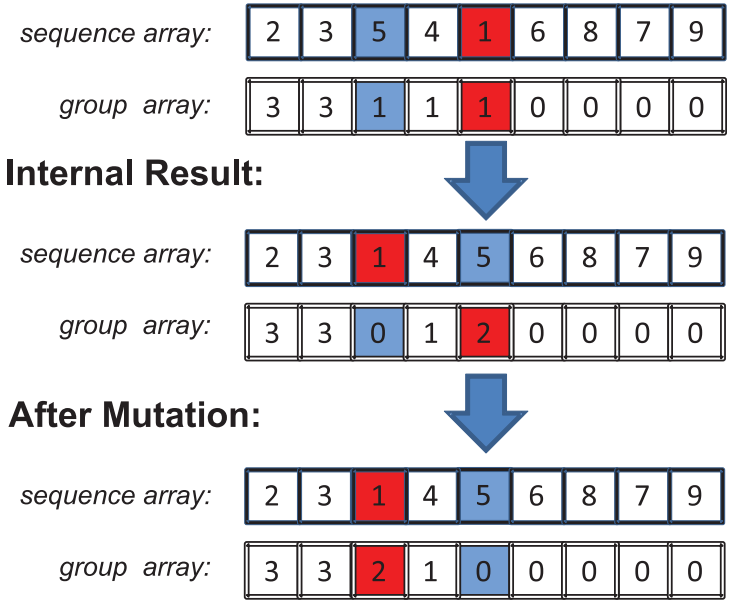

Fig. 4. The Example for Mutation

Fig.4 illustrates an example of the mutations of sequence array and group array. We assume that the third and fifth elements are selected by the random seed. For the sequence array, the elements of " 5 " and "1" change their positions after the mutation of sequence array as shown in the figure. On the other hand, from the aspect of group array, the situation is a bit complicated. After the decrease in third element and the increase in fifth element, the group array shown as "Internal Result" in the figure is not in numerical order. It requires a sorting to produce the final group array. Otherwise, the solution repetition can not be avoided.

3) Selection Operators: For the purpose of selecting the better genes to survive for the evolution at the next round, we propose a fitness function to evaluate the performance of a gene. As to obtain an efficient solution, we employ the same criterion to estimate the communication cost of an itinerary, as in [9].

During the GA evolution, the $i$ th gene corresponds to a planned itinerary, whose cost is denoted by $E_{I}(i), i=1 \ldots k$, where $k$ is the size of the search space. After the crossover and mutations, the number of genes is increased to $(1+\alpha) k{ }^{5}$ In our implementation, the select operator select first $k$ genes according to the their better $E_{I} \mathrm{~s}$.

\section{Simulation}

\section{A. Simulation Setup}

We implemented the proposed GA-MIP algorithm as well as several existing SIP algorithms, e.g., LCF [8] and MADD [7], using OPNET $^{6}$ Modeler and perform simulations. The same network model in [11] is adopted, in which the nodes are uniformly deployed within a $1000 \mathrm{~m} \times 500 \mathrm{~m}$ field, and the sink node is located at the center of the field and multiple source nodes are randomly distributed in the network. To verify the scaling property of our algorithms, we select a large-scale network with 800 nodes. The parameters for MA system are shown in following Table I.

TABLE I

Simulation Parameters For Mobile Agent System

\begin{tabular}{|c|c|}
\hline Raw Data Reduction Ratio & 0.8 \\
\hline Aggregation Ratio & 0.9 \\
\hline Raw Data Size & 2048 bits \\
\hline MA Code Size & 1024 bits \\
\hline MA Accessing Delay & $10 \mathrm{~ms}$ \\
\hline Data Processing Rate & $50 \mathrm{Mbps}$ \\
\hline
\end{tabular}

\section{B. Evaluation Metrics}

In order to evaluate the time and energy efficiency from the simulation results, we consider the following four performance metrics, as several previous work [9] [11]:

- Estimated Cost: The value of estimated energy cost calculated by the fitness function. Even though these values are just approximate energy cost in the network, they still could be a criteria to evaluate the convergence of the GA evolution progress.

- Task Duration: In a SIP algorithm, it is equivalent to average end-to-end report delay, which is the average delay from the time when a MA is dispatched by the sink to the time when the agent returns to the sink. In

\footnotetext{
${ }^{5} \alpha$ is a constant which is set to 1 in our simulation.
}

${ }^{6}$ OPNET, http://www.opnet.com/ 


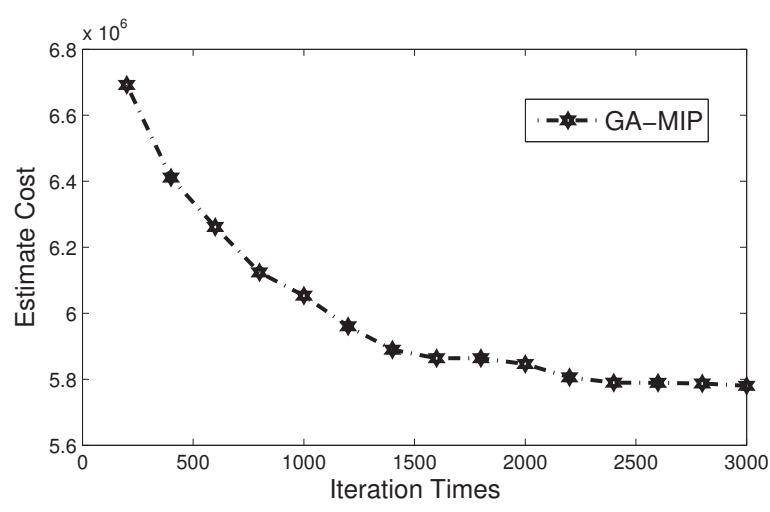

(a) The larger iteration times, the lower estimated cost

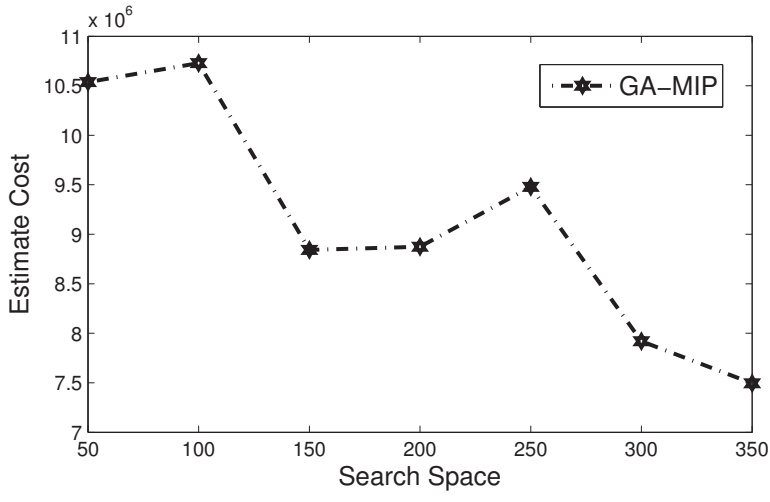

(b) The bigger search space, the lower estimated cost

Fig. 5. Using the larger iteration times and the bigger search space, the lower estimated cost can be obtained

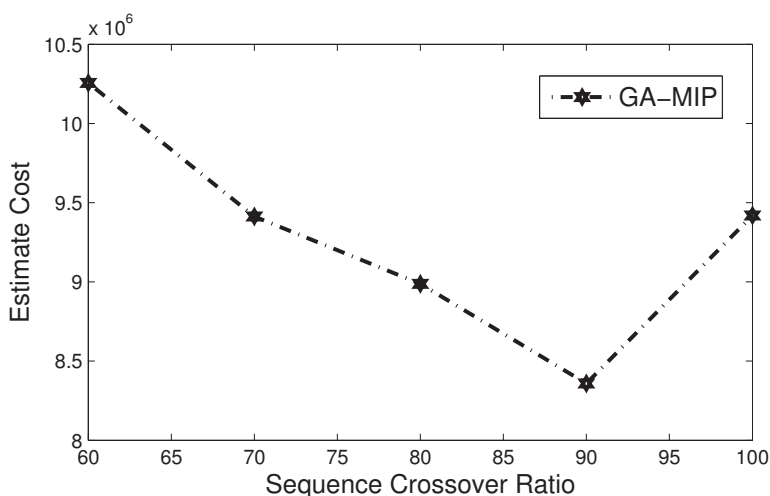

(a) The lowest cost is achieved when the Sequence Crossover Ratio is 0.9

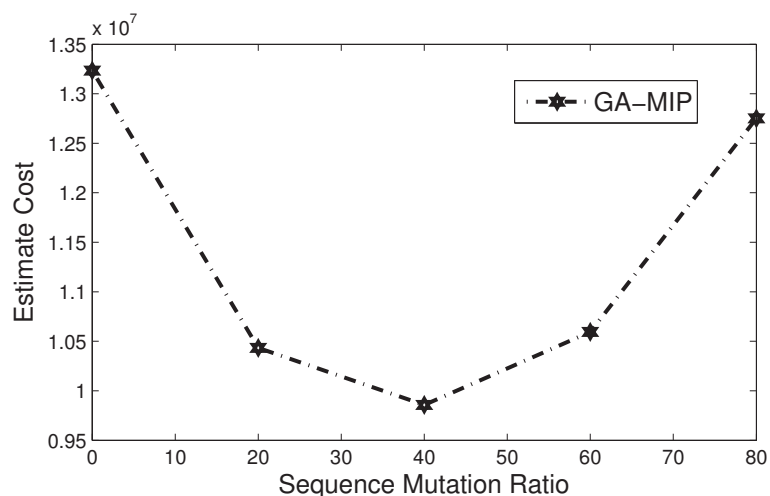

(b) The lowest cost is achieved when the Sequence Mutation Ratio is 0.4

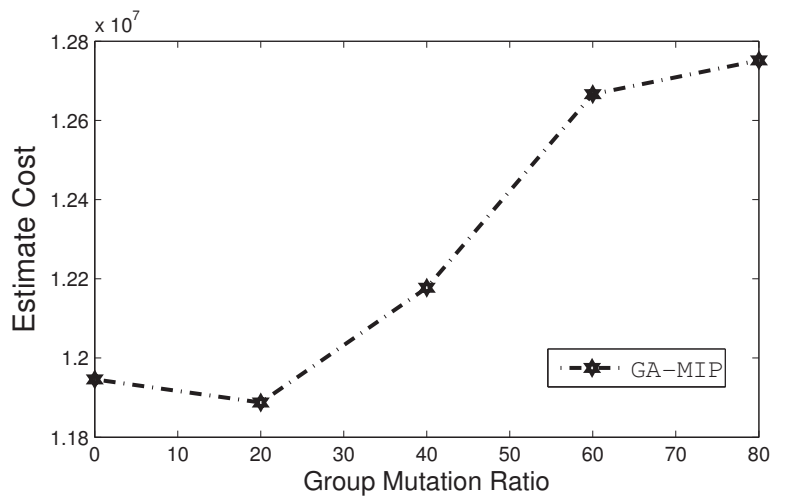

(c) The lowest estimated cost is achieved when Group Mutation Ratio is 0.2

Fig. 6. The impacts of the changing Crossover and Mutation Ratios

an MIP algorithm, since multiple agents work in parallel, there must be one agent which returns to the sink at last. Then, the task duration of an MIP algorithm is the delay of that agent.

- Average Communication Energy: The total communication energy consumption, including transmitting, receiving, retransmissions, overhearing and collision, to obtain each sensory data from all the target sources.

- Energy-Delay Product (EDP): For time-sensitive applications over energy constrained WSNs, EDP (calculated by
$E D P=$ energy $\times$ delay $)$ gives us a unified view. The smaller the value of is, the better the unified performance will be.

\section{Parameters Selection for Genetic Algorithm}

Some parameters should be set up for the genetic algorithm, such as the number of iteration, the size of search space, the ratio of crossover and mutations for sequence array and group array. In this section, we evaluate the impacts of these parameters on the efficiency of the GA evolution. 
As shown in Fig.5, using the larger number of iteration times and the bigger search space, the lower estimated cost can be obtained. However, the more iteration times and the bigger search space will cause higher computational complexity. Thus, there is a tradeoff between the performance and the computational complexity. In the Fig.5, algorithm converges quickly after 1500 iterations. As for the search space, 300 is also enough to achieve a low estimated cost. Fig.6 indicates the best value for crossover and mutation ratios. Base on the information, we set sequence crossover ratio to 0.9 (as shown in Fig.6(a)), sequence mutation ratio to 0.4 (as shown in Fig.6(b)), and group mutation ratio to 0.2 (as shown in Fig.6(c)).

In Table II, the used GA parameters for the following simulations are listed.

TABLE II

Simulation PARAMETERS FOR GA-MIP

\begin{tabular}{|c|c|}
\hline GA Iteration Times & 1500 \\
\hline GA Search Spaces & 300 \\
\hline Sequence Crossover Ratio & 0.9 \\
\hline Sequence Mutation Ratio & 0.4 \\
\hline Grouping Mutation Ratio & 0.2 \\
\hline
\end{tabular}

\section{Visualization of search Result Computed by GA-MIP and $L C F$}

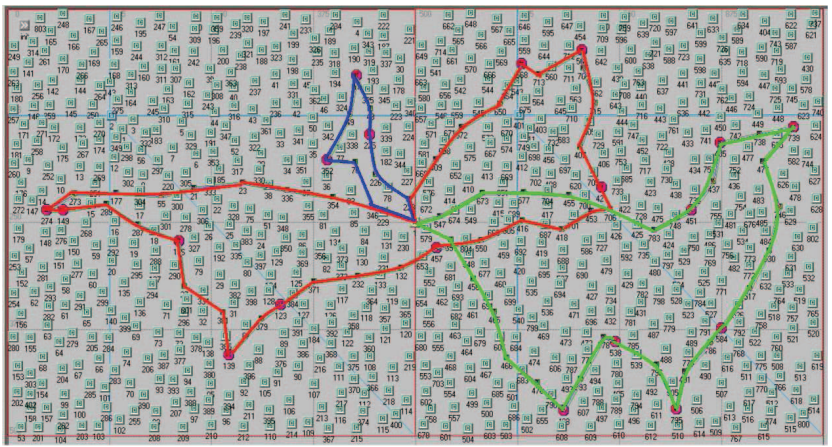

Fig. 7. Visualization of GA-MIP with 3 MAs

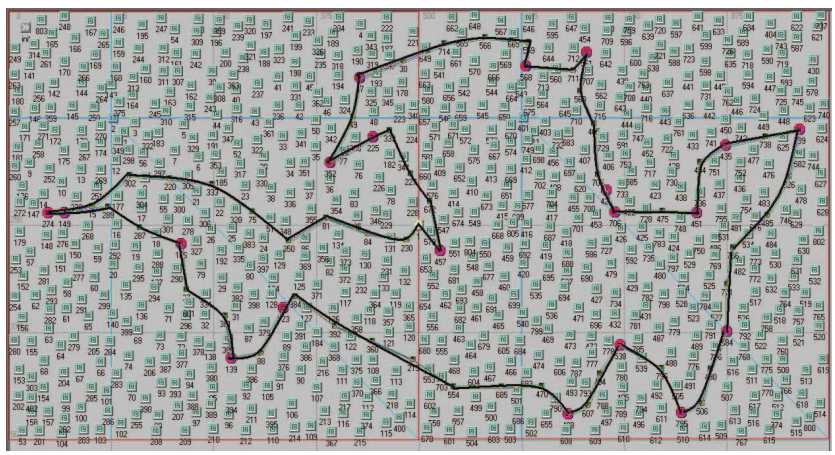

Fig. 8. Visualization of LCF with $1 \mathrm{MA}$

In order to provide the clear impression on the difference of both single MA itinerary planning (SIP) problem and multiple mobile agents itinerary planning (MIP) problem, Figs.7 and 8 are provided in this Subsection. The simulations are made in the previously defined evaluating environment in OPNET with 20 randomly deployed source nodes. Fig.7 shows the result of the itinerary searching for GA-MIP, in which three MAs are sent by the sink node to collect the sensory data in source nodes simultaneously. And, Fig. 8 shows the result of the itinerary searching for LCF, a single MA travels a long route in the network. The task duration is reduced extensively through the data collections by multiple MAs in parallel.

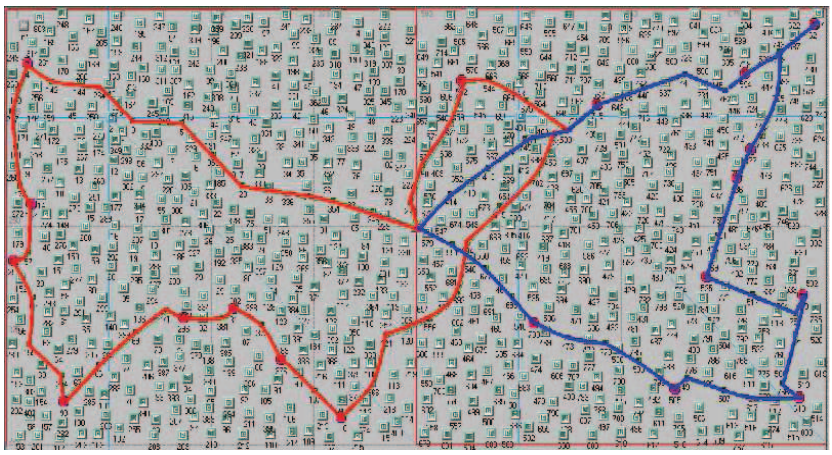

Fig. 9. Visualization of GA-MIP with 2 MAs

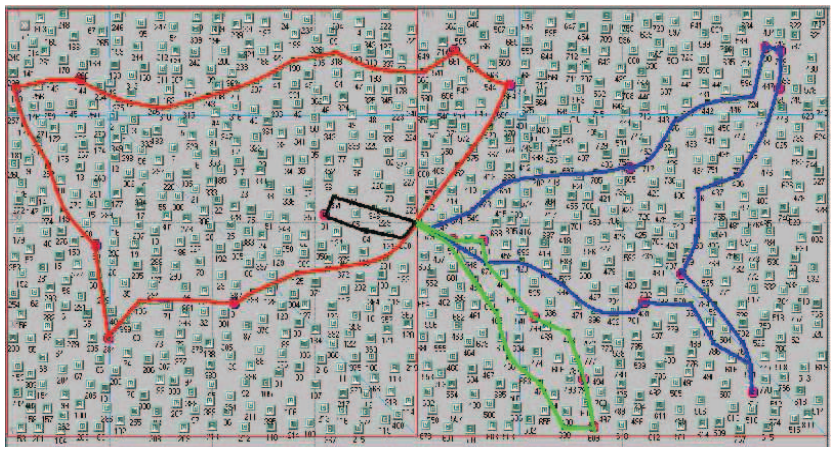

Fig. 10. Visualization of GA-MIP with 4 MAs

We also provide two more snapshots for readers to see the execution results of GA-MIP scheme with different source node deployments. Note, the number of source nodes is still 20. It is easy to see that even though the number of source nodes used in the following two scenarios is the same, but due to the different random deployment, the number of MAs required by the GA-MIP scheme is different. e.g., in Fig.9, only two MAs are needed, but in Fig. 10 four MAs are needed.

\section{E. Performance Comparison of GA-MIP, LCF and MADD with Varying Source Number}

In this Subsection, we compare the GA-MIP scheme with two previous SIP algorithms, i.e., LCF [8] and MADD [7]. During the simulation work, the number of source nodes is changed for 10 to 80 by the step of 10 . For each scenario of particular number of source nodes, extensive simulations are performed with different random seeds. The distribution 
of source nodes is randomly selected by the recalculation of the seeds.

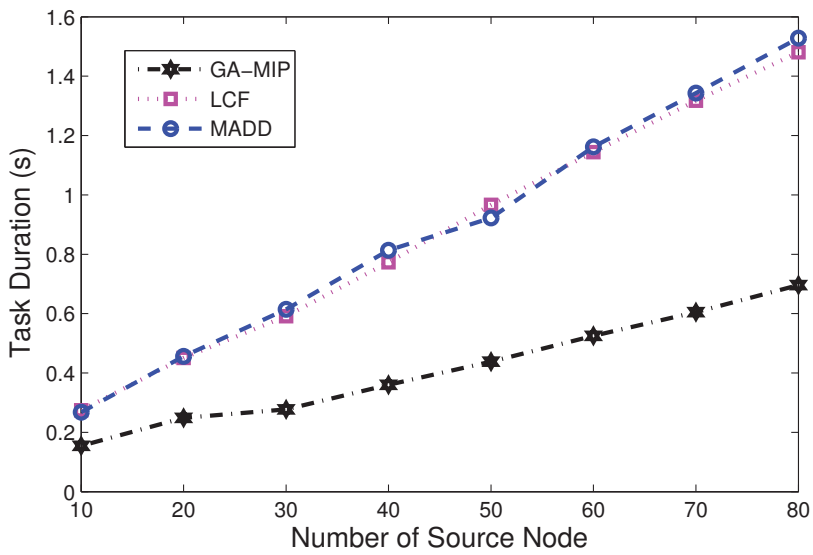

Fig. 11. The impact of number of source nodes on Task Duration

As shown in Fig.11, GA-MIP algorithm has large advantage in terms of task duration, which is only half of that of LCF and MADD. The reason for this phenomenon is that in single MA systems, one MA should travel along the whole network to collect information in all sensor nodes. This procedure will cost a larger latency since the sensor nodes are always distributed all over the network. Multiple MAs can speed up the task because more than two itineraries are applied simultaneously.

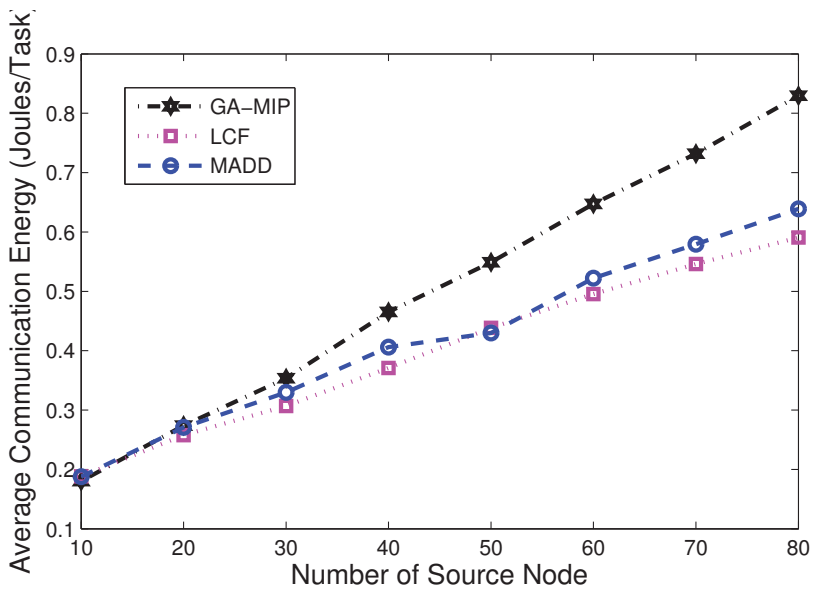

Fig. 12. The impact of number of source nodes on Energy Cost

In Fig.12, the energy consumption of GA-MIP algorithm is a bit higher than that of SIP algorithms. It is because multiple MAs are sent in GA-MIP solution, thus more energy is consumed for delvering the larger size of processing codes while they are traveling in the WSN. However, from the visualization in Figs.7 and 8, we can realize that the MIP solutions cover more intermediate nodes in the dissemination procedure. It implies that MIP can achieve better global load balancing than SIP solutions, thus, achieve a longer network lifetime.

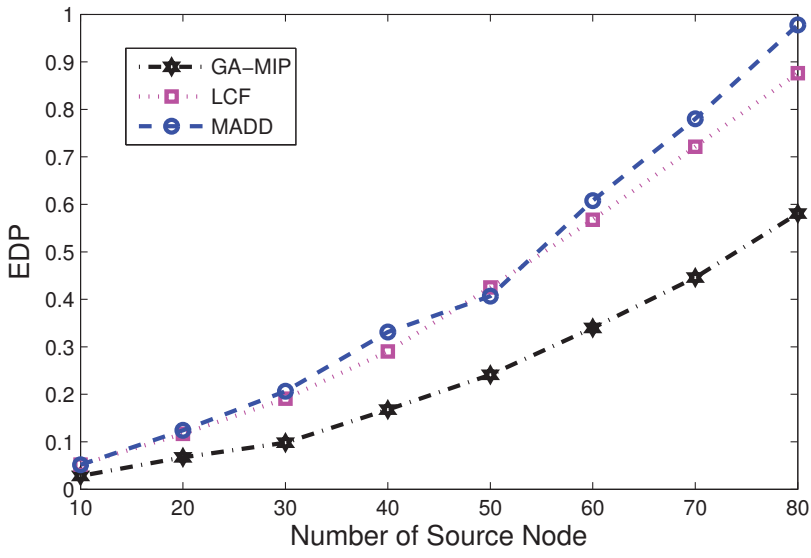

Fig. 13. The impact of number of source nodes on EDP

However, the integrated performance of delay and energy (i.e., EDP) is an important overall criterion in many applications, especially for delay constraint traffic in wireless sensor network, such as wireless multimedia sensor network, and video sensor networks. In Fig.13, we compares the EDP for GA-MIP and other SIP algorithms. It shows that GA-MIP algorithm achieves the best overall performance among the three schemes, which verifies effectiveness of the proposed algorithm.

\section{CONCLUSION}

Applying MAs in WSNs can facilitate a large number of location aware applications. In this paper, we first investigate several existing SIP solutions to indicate the shortcoming of using MA, such as larger latency and global unbalancing in large scale WSNs. These challenging issues need to be addressed to enable MA based systems being deploying to a wide range of applications in WSN. Thus, we proposed a Genetic Algorithm based Multi-Mobile Agents itinerary planning (GA-MIP) to address the problems. The use of GA approach Extensive simulations have been performed to show the better integrated performance of GA-MIP. For the future work, we are going to further investigate MIP problem in follow two more complicated network scenarios:

- Mobile Sink Node [17]: We can see that if the sink node can be mobile, and it can autonomously move inside the network field of the WSN, there is a good opportunity that we can further minimize the task duration and total communication cost by moving the sink node to a optimal location in the WSN.

- Duty Cycled WSNs [18]: Currently, we assume that all the sensor nodes are working for all the time during the studying of the problem, which actually is not feasible in the real WSNs applications. We are going to further investigate the MIP problem when some of sensor nodes are turned into sleep. In other words, we are going to further study the impacts on MIP problem brought by the changing of the WSNs node density. 


\section{ACKNOWLEDGEMENT}

This research was partially supported by Grant-in-Aid for Scientific Research (S)(21220002) of the Ministry of Education, Culture, Sports, Science and Technology, Japan; and partially supported by the Lion project supported by Science Foundation Ireland under grant no. SFI/02/CE1/I131.

\section{REFERENCES}

[1] K. Romer and F. Mattern, "The design space of wireless sensor networks," IEEE Wireless Communications, vol. 11, no. 6, 2004.

[2] L. Tong, Q. Zhao, and S. Adireddy, "Sensor networks with mobile agents," in Proceedings of the 2003 IEEE International Conference on Military Communications (MILCOM 2003), Boston, Massachusetts, USA, 2003.

[3] M. Beigl, A. Krohn, T. Zimmer, C. Decker, and P. Robinson, "AwareCon: situation aware context communication," in Proceedings of the 5th IEEE International Conference on Ubiquitous Computing (UbiComp 2003), Seattle, Washington, USA, 2003, pp. 132-139.

[4] L. Shu, Y. Zhang, Z. Yu, L. T. Yang, M. Hauswirth, and N. Xiong, "Context-aware cross-layer optimized video streaming in wireless multimedia sensor networks," The Journal of Supercomputing, Springer, 2009.

[5] M. Chen, S. Gonzalez, and V. Leung, "Applications and design issues for mobile agents in wireless sensor networks," Wireless Communications, IEEE, vol. 14, no. 6, pp. 20-26, 2007.

[6] M. Chen, T. Kwon, Y. Yuan, and V. C. Leung, "Mobile agent based wireless sensor networks," Journal of Computers, vol. 1, no. 1, 2006.

[7] M. Chen, T. Kwon, Y. Yuan, Y. Choi, and V. C. M. Leung, "Mobile agent-based directed diffusion in wireless sensor networks," EURASIP J. Appl. Signal Process., vol. 2007, no. 1, pp. 219-219, 2007.

[8] H. Qi and F. Wang, "Optimal itinerary analysis for mobile agents in ad hoc wireless sensor networks," in Proceedings of the IEEE 2001 International Conference on Communications (ICC 2001), Helsinki, Finland, 2001.

[9] M. Chen, V. Leung, S. Mao, T. Kwon, and M. Li, "Energy-Efficient itinerary planning for mobile agents in wireless sensor networks," in Proceedings of the IEEE 2009 International Conference on Communications (ICC 2009), Bresden, Germany, 2009, pp. 1-5.

[10] Q. Wu, N. S. V. Rao, J. Barhen, S. S. Iyengar, V. K. Vaishnavi, H. Qi, K. Chakrabarty, S. Member, and S. Member, "On computing mobile agent routes for data fusion in distributed sensor networks," IEEE Trans. Knowledge and Data Engineering, vol. 16, pp. 740-753, 2004.

[11] M. Chen, S. Gonzlez, Y. Zhang, and V. C. Leung, "Multi-agent itinerary planning for sensor networks," in Proceedings of the IEEE 2009 International Conference on Heterogeneous Networking for Quality, Reliability, Security and Robustness (QShine 2009), Las Palmas de Gran Canaria, Spain, 2009.

[12] R. Szewczyk, A. Mainwaring, J. Polastre, J. Anderson, and D. Culler, "An analysis of a large scale habitat monitoring application," in Proceedings of the ACM 2004 2nd International Conference on Embedded Networked Sensor Systems (SenSys 2004), Boston, MA, USA, 2004, pp. 214-226.

[13] L. Shu, C. Wu, Y. Zhang, J. Chen, L. Wang, and M. Hauswirth, "Nettopo: beyond simulator and visualizer for wireless sensor networks," ACM SIGBED Review, vol. 5, no. 3, 2008.

[14] M. Mitchell, An Introduction to Genetic Algorithms, 1998. MIT Press.

[15] R. Poli and W. B. Langdon, "Schema theory for genetic programming with One-Point crossover and point mutation," Evolutionary Computation, vol. 6, no. 3, pp. 231-252, 1998.

[16] K. A. D. Jong and W. M. Spears, "A formal analysis of the role of multi-point crossover in genetic algorithms," Annals of Mathematics and Artificial Intelligence, vol. 5, no. 1, pp. 1-26, Mar 1992.

[17] D. W. W. L. H. K. W. YanWang Zhao, Qianping Jiang, "An agent-based routing protocol with mobile sink for wsn in coal mine," in Proceedings of the 3rd International Conference on Pervasive Computing and Applications (ICPCA 2008),, Alexandria, Egypt, 2008.

[18] L. Cheng, C. Chen, J. Ma, L. Shu, H. Chen, and L. T. Yang, "Residual time aware forwarding for randomly duty-cycled wireless sensor networks," in Proceedings of the 7th IEEE/IFIP International Conference on Embedded and Ubiqutious Computing (EUC 2009),, Vancouver, Canada, 2009 